\title{
L'étude de la croissance de Oreochromis niloticus par la fertilisation des étangs : le cas de la ferme Naviundu Lubumbashi
}

\author{
Ipungu L1, Ngoy $\mathrm{K}^{1}$, Banze $\mathrm{K}^{2}$, Lumfwa $\mathrm{K}^{2}$, Kafund $\mathbf{M}^{1}$ \\ ${ }^{1}$ Service de Pisciculture, Pathologie des Poissons et Gestion des Ressources Halieutiques Fac. Med. Vet. UNILU. \\ BP 1825 R.D Congo \\ 2Service de Parasitologie et Maladies Parasitaires, Fac. Med. Vet. UNILU. BP 1825 R.D Congo. \\ Corresponding author email : ipunguraymond@yahoo.fr, kmarcelngoy@gmail.com, i.banze@hotmail.com, \\ klumfwa@yahoo.com
}

Original submitted in on $12^{\text {th }}$ June 2015. Published online at www.m.elewa.org on $31^{\text {st }}$ July 2015 http://dx.doi.org/10.4314/jab.v91i1.3

\section{RESUME}

Objectif : Les auteurs ont étudié la croissance de Oreochromis niloticus en fonction de la fertilisation des étangs à base des crottes des chèvres et des excréments des porcs à la ferme Naviundu...

Méthodologie et résultats : L'étude a porté sur 90 alevins de Oreochromis niloticus dont le poids initial a été de $2 \mathrm{~g}$. Au bout de 3 mois d'étude, différents poids moyens ont été obtenus : $52,1 \mathrm{~g}$ avec les excréments des porcs ; $21,9 \mathrm{~g}$ avec les crottes des chèvres et $8,03 \mathrm{~g}$ pour l'étang témoin.

Conclusion et interprétation des résultats : En faisant recours au test statistique et aux analyses de laboratoire, les résultats obtenus montrent qu'il y a une différence significative entre les poids des poissons, il ressort que les engrais ont un impact positif sur les poids des poissons. La productivité des eaux dépend de la quantité d'éléments fertilisants apportés à l'étang et des facteurs particuliers à l'étang : la nature du fond, des sédiments, la turbidité, la composition chimique de l'eau, la charge en poisson et la température.

Mots-clés : Croissance, Oreochromis niloticus, Fertilisation, Étang, Lubumbashi.

\begin{abstract}
Objective : The growth of Oreochromis niloticus by pond fertilization using goat droppings and swine excrement was studied in Naviundu farm.

Methodology and results: The study involved 90 Oreochromis niloticus fingerlings with initial weight of $2 \mathrm{~g}$. After 3 months of study, different mean weights were obtained: $52.1 \mathrm{~g}$ with the faeces of pigs; $21.9 \mathrm{~g}$ with the droppings of goats and $8.03 \mathrm{~g}$ for the control pond.

Conclusion and results interpretation: By making use of statistical testing and laboratory analysis, results showed that there is a significant difference between the weights of the fish. It appears that fertilizers have a positive impact on the weight of the fish. The productivity of water depends on the amount of nutrients brought to the pond and the pond-specific factors: the nature of the bottom sediment, turbidity, chemical composition of the water, the fish over, and temperature.
\end{abstract}

Keywords : Growth, Oreochromis niloticus, fertilization, Pond, Lubumbashi. 


\section{INTRODUCTION}

Pour assurer une bonne physiologie humaine, tout peuple en général, et peuple congolais en particulier a besoin d'un équilibre alimentaire. L'une des règles élémentaires en nutrition consiste à maintenir l'adéquation entre les besoins en nutriments et les apports alimentaires. II nécessite un apport considérable et régulier en protéines animales (Feillet, 2014). La chair de poisson est un aliment le plus apprécié, moins couteux et accessible à toutes les couches sociales congolaises en particulier et du monde en général (Carballo et al., 2008 ; Amakoe, 2011 ; Anonyme, 2007). Vu les besoins toujours grandissants de la consommation des poissons dans la population, une information sur la recherche d'améliorer la pisciculture s'avère nécessaire. Cette préoccupation a suscité l'entreprise d'une étude de la croissance de Oreochromis niloticus (Tilapia nilotica) en fonction de la fertilisation des étangs en utilisant les engrais organiques des élevages. Les engrais sont les matériels ajoutés à l'étang pour rendre celui-ci plus productif, c'est notamment le matériel végétal transformé en compost ou du fumier animal. (Hilbrands et Yzerman, 2004). On peut citer pour le matériel végétal ; les choux, les feuilles de manioc et pour le fumier animal ; les excréments de porc, les crottes de chèvres, lls permettent à l'étang de fournir les éléments nécessaires soit directement aux poissons soit aux planctons qui sont la source majeure des aliments naturels pour les poissons (Hilbrands et Yzerman, 2004; Chambres
d'Agriculture, 2005). Ils doivent être en quantité suffisante (car les fumiers des animaux sont habituellement appliqués aux étangs selon un rapport poids par surface d'étang ou selon un rapport animal par surface) et de bonne qualité (Hilbrands et Yzerman, 2004; Chambres d'Agriculture de Lorraine 2005 ; Carballo et al., 2008 ; Wurtz-Arlet, 1980). La croissance est un processus biologique complexe qui fait intervenir des nombreux facteurs (Melard, 1986). C'est une étape dynamique de la vie pendant laquelle l'être nait, croit et meurt (Debaisieux, 1946, cité par Kestemont et al ,2004). Oreochromis niloticus, espèce thermophile, se rencontre en milieu naturel entre $14^{\circ}$ et $35^{\circ} \mathrm{C}$. Ce poisson est résistant (peut supporter des températures de $7{ }^{\circ} \mathrm{C}$ à $41^{\circ} \mathrm{C}$ et une salinité de 0,015 à 30 pour mille et un pH de 5 à 11), il vit bien dans des eaux de basse qualité, les eaux douces tropicales et subtropicales. Et il se reproduit facilement (Hilbrands et Yzerman, 2004, Amakoe, 2011). Oreochromis niloticus est reconnu pour sa croissance rapide (Amaoke, 2011), sa durée de vie est relativement courte (4-7ans), sa vitesse de croissance est relativement variable selon les milieux. A cet effet, la Pisciculture constitue une source non négligeable en protéines animales, facile à exploiter dans notre pays dont la potentialité en eau est incontestable.

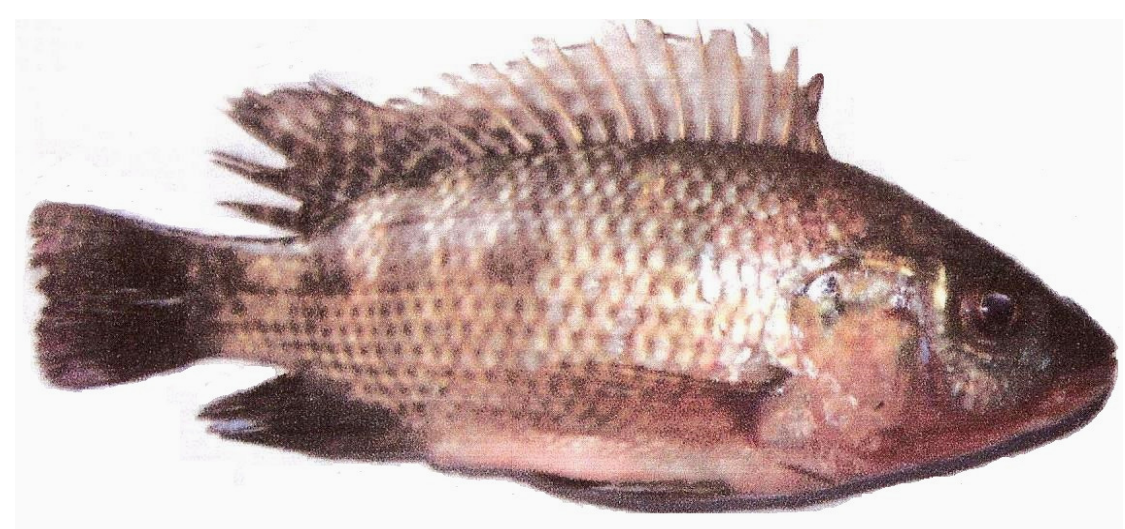

Figure1: Tilapia nilotica (Oreochromis niloticus) /NAVIUNDU 

étangs: le cas de la ferme Naviundu Lubumbashi

\section{MILIEU, ANIMAUX, MATERIEL ET METHODES}

MILIEU : Des investigations ont eu lieu à la ferme Naviundu de février à avril, 2011. La ferme Naviundu est située dans la partie méridionale de la province du Katanga en République Démocratique du Congo à $27^{\circ} 28^{\prime}$ de longitude Est et 11040' de latitude sud, à une altitude moyenne de plus au moins $1230 \mathrm{~m}$, dans la commune annexe à $8 \mathrm{~km}$ de la ville de Lubumbashi. La classification de KOPPEN nous montre que Lubumbashi est établi dans une région au climat de type CW7 caractérisé par alternance de deux saisons a durée inégale et séparée par une petite période qui marque l'intersaison, suite à sa position entre l'équateur et les tropiques de Capricorne. Le sol appartient en grande partie aux groupes de sols ferralitiques rouges, le terrain est peu perméable à la ferme Naviundu. La végétation est du type savane arbuste, constituée des graminées, des légumineuses et de certaines plantes toxiques.

ANIMAUX: Cette étude a porté sur 90 alevins de Oreochromis nilotcus d'origine Shirundu provenant de la station d'aquaculture de Kipopo, dont le poids moyen était de $2 \mathrm{~g}$. Ces poissons ont été élevés dans un système intensif au site précité pendant une période allant du mois de Février au mois d'Avril 2011.

METHODES : Le dispositif expérimental a utilisé 3 étangs dans lesquels étaient repartis 30 alevins par étang, de même poids, de sorte que chacun subit un traitement diffèrent de l'autre, le premier recevant les excréments de porc, le second les crottes de chèvre et le troisième rien, car il joue le rôle de l'étang témoin.
Épandage du fumier :Chaque jour, le fumier a été distribué dans l'étang en fonction de la qualité de l'eau et de la biomasse des alevins y compris le comportement de poissons, soit en augmentant progressivement cette quantité ou en la diminuant. Les quantités suivantes de fumier ont été utilisées

- Excrément des porcs: un étang de $50 \mathrm{~m}^{2}$ $=50 \times 6 / 100=3 \mathrm{~kg} / \mathrm{J}$

-Crotte des chèvres : un étang de $50 \mathrm{~m}^{2}=50 \times 4 / 100=2 \mathrm{~kg} / \mathrm{J}$ La composition en éléments nutritifs des excréments de porcs et de chèvres, selon Kestemont et al. (2004), est la suivante :

- Excrément de porc :

- Crotte de chèvre :

$\mathrm{N}=2,04$;

$\mathrm{N}=3,03$

$P=0,73$

$P=1,66$

$\mathrm{K}=0,47$

$\mathrm{K}=1,60$

Prélèvement des paramètres physico-chimiques des étangs

Les paramètres physiques de l'eau

Prélèvement de la transparence : Pour la prise de la transparence, un disque de Secchi peint en quadrants blanc et noir, et attache à un mètre en bois gradué de 0 à $45 \mathrm{~cm}$ a été utilisé. Par le mètre gradué, on laisse descendre le disque et on note la profondeur à laquelle, il disparait et on note la profondeur à sa réapparition à l'œil de l'observateur.

Prélèvement de la température : La température est l'élément physique très important gouvernant les activités du poisson. Elle est prélevée à l'aide d'un thermomètre à mercure.

Tableau 1 : Résumé des températures prélevées aux mois de Février, Mars et Avril

\begin{tabular}{l|l|l|l|l}
\hline No & Mois & Température moyenne mensuelle & Température max & Température min \\
\hline $\mathbf{1}$ & Février & $22,6^{\circ} \mathrm{C}$ & $25^{\circ} \mathrm{C}$ & $20^{\circ} \mathrm{C}$ \\
\hline $\mathbf{2}$ & Mars & $23,13^{\circ} \mathrm{C}$ & $26^{\circ} \mathrm{C}$ & $20^{\circ} \mathrm{C}$ \\
\hline $\mathbf{3}$ & Avril & $23,9^{\circ} \mathrm{C}$ & $26^{\circ} \mathrm{C}$ & $20^{\circ} \mathrm{C}$ \\
\hline
\end{tabular}

Prélèvement du pH : Pour prélever le pH de nos étangs d'expérimentation, on mettait de l'eau dans un récipient propre pour les analyses de laboratoire. L'eau des étangs a donné un $\mathrm{pH}$ de 6,8 pour l'ensemble du temps de notre étude.

Les paramètres chimiques: Les échantillons d'eau prélevés dans les étangs d'expérimentation ont été analysés à la faculté des sciences de l'Université de Lubumbashi. Les résultats des analyses ont révélé les valeurs suivantes:

$-\mathrm{NO}_{3}: 0,005 \mathrm{mg} / \mathrm{l}$

$-\mathrm{NO}_{2}: 0,07 \mathrm{mg} / \mathrm{l}$

$-\mathrm{O}_{2}: 3,53 \mathrm{ppm}$
-Carbonate: 302, 2 mg/l

-Bicarbonate: $293 \mathrm{mg} / \mathrm{l}$

Fertilisation des étangs avec les engrais organiques Fertilisation avec les excréments de porc : L'examen de la situation du mois de Février démontre qu'il y a une faible moyenne au premier prélèvement mais élevée au quatrième prélèvement. L'examen du mois de Mars montre que la moyenne au quatrième prélèvement est élevée et plus ou moins faible au premier prélèvement. Au regard de la situation du mois d'Avril, il a été constaté une moyenne inférieure au premier prélèvement et élevée au quatrième. 
Ipungu et al. J. Appl. Biosci. L'étude de la croissance de Oreochromis niloticus par la fertilisation des étangs: le cas de la ferme Naviundu Lubumbashi

Tableau 2 : croissance de 30 alevins de Oreochromis niloticus (fertilisation aux crottes de porc)

\begin{tabular}{|c|c|c|c|c|c|c|c|c|c|c|c|c|}
\hline Mois & $\begin{array}{l}\text { PT 1er } \\
\text { PLV }\end{array}$ & $\begin{array}{l}\text { PT } 2^{\mathrm{e}} \\
\text { PLV }\end{array}$ & $\begin{array}{l}\text { PT } 3^{\mathrm{e}} \\
\text { PLV }\end{array}$ & $\begin{array}{l}\text { PT 4e } \\
\text { PLV }\end{array}$ & $\begin{array}{l}\text { Pm 1 } \\
\text { PLV }\end{array}$ & $\begin{array}{l}\text { Pm 2 } \\
\text { PLV }\end{array}$ & $\begin{array}{l}\text { Pm } 3^{\mathrm{e}} \\
\text { PLV }\end{array}$ & $\begin{array}{l}\text { Pm } 4^{\mathrm{e}} \\
\text { PLV }\end{array}$ & $\begin{array}{l}\delta 1^{1} \text { er } \\
\text { PLV }\end{array}$ & $\begin{array}{l}\delta 2^{\mathrm{e}} \\
\mathrm{PLV}\end{array}$ & $\begin{array}{l}\delta \quad 3^{e} \\
\text { PLV }\end{array}$ & $\begin{array}{l}\delta \quad 4^{e} \\
P L V\end{array}$ \\
\hline Février & 214 & 361 & 476 & 630 & 7,13 & 12,03 & 15,86 & 21 & 1,35 & 1,42 & 1,52 & 1,83 \\
\hline Mars & 784 & 908 & 1034 & 1145 & 26,13 & 30,26 & 34,46 & 38,16 & 2,44 & 2,22 & 2,55 & 2,22 \\
\hline Avril & 1233 & 1313 & 1402 & 1563 & 41,1 & 43,76 & 46,73 & 52,1 & 2,83 & 2,53 & 1,65 & 2,24 \\
\hline
\end{tabular}

Légende : PT : Poids total des alevins en gramme ; Pm : Poids moyen des alevins en gramme ; $\delta$ : Écart-type ; PLV :Prélèvement

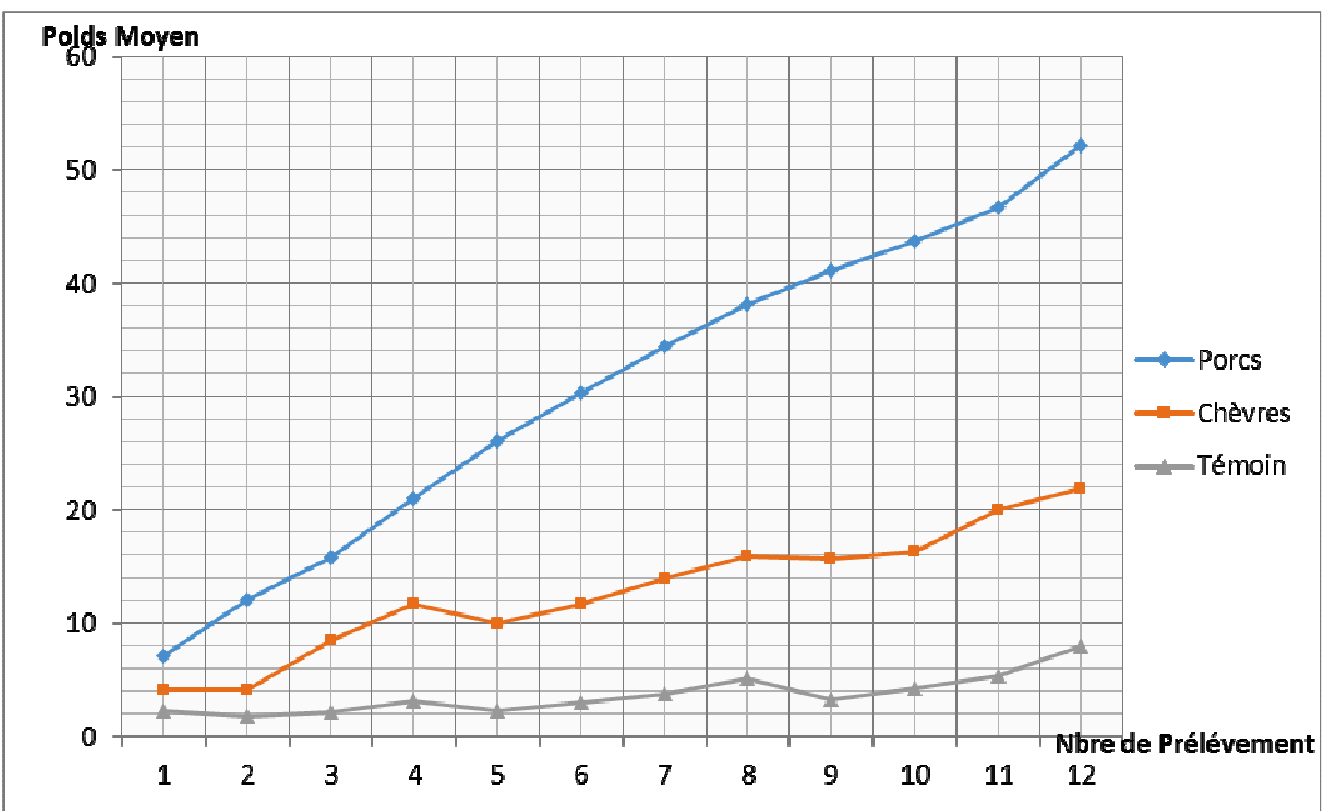

Figure 2 : Courbe de tendance de moyennes (Excrément des porcs, crottes des chèvres et témoin)

L'examen de la figure 2, démontre que la croissance de Oreochromis niloticus en poids dans les étangs fertilisés avec les excréments de porc qui va de $7,13 \mathrm{~g}$ au premier prélèvement à $52,1 \mathrm{~g}$ au dernier prélèvement est positive. En outre, la croissance de Oreochromis niloticus en poids dans les étangs fertilisés avec les excréments de chèvre est bonne jusqu'au $4^{\mathrm{e}}$ prélèvement, puis baisse au $5^{\mathrm{e}}$ et ensuite croit jusqu'au $12^{e}$ prélèvement. L'examen de la courbe de tendance des moyennes dans l'étang témoin montre que le poids moyen n'est pas constant, il varie de 0,83 à $3 \mathrm{~g}$ par prélèvement (Tableau 4).
Fertilisation avec les crottes de chèvre : Au regard de la situation du mois de Février, nous avons constaté une faible moyenne au $1^{\text {er }}$ prélèvement et elle croit progressivement au quatrième prélèvement. L'examen de la situation du mois de Mars montre que, la moyenne au quatrième prélèvement est plus élevée qu'à celle $d u$ premier. Au regard de la situation du mois d'avril, il a été remarqué une faible moyenne au 1 er prélèvement et élevé au 4 e prélèvement (Tableau 3).

Tableau 3 : croissance de 30 alevins de Oreochromis niloticus après fertilisation au moyen des crottes de chèvre

\begin{tabular}{|c|c|c|c|c|c|c|c|c|c|c|c|c|}
\hline Mois & $\begin{array}{l}\text { PT 1er } \\
\text { PLV }\end{array}$ & $\begin{array}{l}\text { PT 2e } \\
\text { PLV }\end{array}$ & $\begin{array}{l}\text { PT 3e } \\
\text { PLV }\end{array}$ & $\begin{array}{l}\text { PT 4 } \\
\text { PLV }\end{array}$ & $\begin{array}{l}\text { Pm 1 } 1^{\text {er }} \\
\text { PLV }\end{array}$ & $\begin{array}{l}\text { Pm } 2^{\mathrm{e}} \\
\text { PLV }\end{array}$ & $\begin{array}{l}\text { Pm } 3^{e} \\
\text { PLV }\end{array}$ & $\begin{array}{l}\text { Pm 4e } \\
\text { PLV }\end{array}$ & $\begin{array}{l}\delta 1^{\text {er }} \\
\text { PLV }\end{array}$ & $\begin{array}{l}\delta \quad 2^{\mathrm{e}} \\
\mathrm{PLVV}\end{array}$ & $\begin{array}{l}\delta \quad 3^{e} \\
\text { PLV }\end{array}$ & $\begin{array}{l}\delta 4^{\mathrm{e}} \\
\mathrm{PLV}\end{array}$ \\
\hline Févrie & 126 & 126 & 256 & 352 & 4,2 & 4,2 & 8, & 11,73 & 1,86 & 1,86 & 2,01 & 1,83 \\
\hline Mars & 300 & 351 & 419 & 479 & 10 & 11,7 & 13,96 & 15,96 & 2,62 & 2,64 & 2,63 & 1,73 \\
\hline Avril & 472 & 489 & 601 & 657 & 15,73 & 16,3 & 20,03 & 21,9 & 5,26 & 3,84 & 4,17 & 4,12 \\
\hline
\end{tabular}

Légende : PT : Poids total des alevins en gramme ; Pm : Poids moyen des alevins en gramme $; \delta$ : Écart-type ; PLV : Prélèvement 
Ipungu et al. J. Appl. Biosci. L'étude de la croissance de Oreochromis niloticus par la fertilisation des étangs: le cas de la ferme Naviundu Lubumbashi

\section{Étang témoin}

Tableau 4 : croissance de Oreochromis niloticus dans l'étang témoin

\begin{tabular}{|c|c|c|c|c|c|c|c|c|c|c|c|c|}
\hline Mois & $\begin{array}{l}\text { PT 1er } \\
\text { PLV }\end{array}$ & $\begin{array}{l}\text { PT 2e } \\
\text { PLV }\end{array}$ & $\begin{array}{l}\text { PT 3e } \\
\text { PLV }\end{array}$ & $\begin{array}{l}\text { PT } 4^{e} \\
\text { PLV }\end{array}$ & $\begin{array}{l}\text { Pm 1er } \\
\text { PLV }\end{array}$ & $\begin{array}{l}\text { Pm 2e } \\
\text { PLV }\end{array}$ & $\begin{array}{l}\text { Pm } 3^{e} \\
\text { PLV }\end{array}$ & $\begin{array}{l}\text { Pm 4e } \\
\text { PLV }\end{array}$ & $\begin{array}{l}\delta 1^{\text {er }} \\
\text { PLVV }\end{array}$ & $\begin{array}{l}\delta \quad 2^{\mathrm{e}} \\
\mathrm{PLV}\end{array}$ & $\begin{array}{l}\delta \quad 3^{e} \\
\text { PLV }\end{array}$ & $\begin{array}{l}\delta 4^{\mathrm{e}} \\
\mathrm{PLV}\end{array}$ \\
\hline Février & 69 & 55 & 66 & 96 & 2,3 & 1,83 & 2,2 & 3,2 & 1,17 & 0,83 & 1,15 & 1,56 \\
\hline Mars & 69 & 90 & 115 & 155 & 2,3 & 3 & 3,83 & 5,16 & 1,23 & 1,81 & 2,50 & 3,18 \\
\hline Avril & 98 & 129 & 161 & 241 & 3,26 & 4,3 & 5,36 & 8,03 & 1,63 & 3,03 & 3,10 & 3,79 \\
\hline
\end{tabular}

Légende : PT : Poids total des alevins en gramme ; Pm : Poids moyen des alevins en gramme $; \delta$ : Écart-type ; PLV : Prélèvement

Tableau 5 : évolution de poids des alevins par semaine en fonction des fertilisants (excrément de porc, crotte de chèvre) et dans l'étang témoin

\begin{tabular}{|c|c|c|}
\hline Semaine & Excrément de (Animal) & Poids \\
\hline 1 & Porc & 7,13 \\
\hline 1 & Chèvre & 4,2 \\
\hline 1 & Témoin & 2,3 \\
\hline 2 & Porc & 12 \\
\hline 2 & Chèvre & 4,2 \\
\hline 2 & Témoin & 1,8 \\
\hline 3 & Porc & 15,9 \\
\hline 3 & Chèvre & 8,5 \\
\hline 3 & Témoin & 2,2 \\
\hline 4 & Porc & 21 \\
\hline 4 & Chèvre & 11,7 \\
\hline 4 & Témoin & 3,2 \\
\hline 5 & Porc & 26,1 \\
\hline 5 & Chèvre & 10 \\
\hline 5 & Témoin & 2,3 \\
\hline 6 & Porc & 30,3 \\
\hline 6 & Chèvre & 11,7 \\
\hline 6 & Témoin & 3 \\
\hline 7 & Porc & 34,5 \\
\hline 7 & Chèvre & 14 \\
\hline 7 & Témoin & 3,8 \\
\hline 8 & Porc & 38,2 \\
\hline 8 & Chèvre & 16 \\
\hline 8 & Témoin & 5,2 \\
\hline 9 & Porc & 41,1 \\
\hline 9 & Chèvre & 15,7 \\
\hline 9 & Témoin & 3,3 \\
\hline 10 & Porc & 43,8 \\
\hline 10 & Chèvre & 16,3 \\
\hline 10 & Témoin & 4,3 \\
\hline 11 & Porc & 46,7 \\
\hline 11 & Chèvre & 20 \\
\hline 11 & Témoin & 5,4 \\
\hline 12 & Porc & 52,1 \\
\hline 12 & Chèvre & 21,9 \\
\hline 12 & Témoin & 8,03 \\
\hline
\end{tabular}


Tableau 6 : Poids moyen des alevins au cours de 12 semaines selon la fertilisation des étangs et dans l'étang témoin

\begin{tabular}{l|l|l|l}
\hline SEMAINE & Étang témoin & Crotte de chèvre & Excrément de porc \\
\hline $\mathbf{1}$ & 2,3 & 4,2 & 7,1 \\
\hline $\mathbf{2}$ & 1,8 & 4,2 & 12 \\
\hline $\mathbf{3}$ & 2,2 & 8,5 & 15,9 \\
\hline $\mathbf{4}$ & 3,2 & 11,7 & 21 \\
\hline $\mathbf{5}$ & 2,3 & 10 & 26,1 \\
\hline $\mathbf{6}$ & 3 & 11,7 & 30,3 \\
\hline $\mathbf{7}$ & 3,8 & 14 & 34,5 \\
\hline $\mathbf{8}$ & 5,2 & 16 & 38,2 \\
\hline $\mathbf{9}$ & 3,3 & 15,7 & 41,1 \\
\hline $\mathbf{1 0}$ & 4,3 & 16,3 & 43,8 \\
\hline $\mathbf{1 1}$ & 5,4 & 20 & 46,7 \\
\hline $\mathbf{1 2}$ & 8 & 21,9 & 52,1 \\
\hline
\end{tabular}

La corrélation entre l'étang témoin et la fertilisation à la crotte de chèvre est de 0,8872 : c'est une corrélation positive. La corrélation entre le témoin et la fertilisation aux excréments de porc est de 0,8349: c'est une corrélation positive. Le coefficient de détermination $\mathrm{R}^{2}$ entre le témoin et la fertilisation au moyen des crottes de chèvre est de 0,787: c'est une régression linéaire positive. Le coefficient de détermination $\mathrm{R}^{2}$ entre le témoin et la fertilisation au moyen des excréments de porc est de 0.697 : c'est une régression linéaire positive.

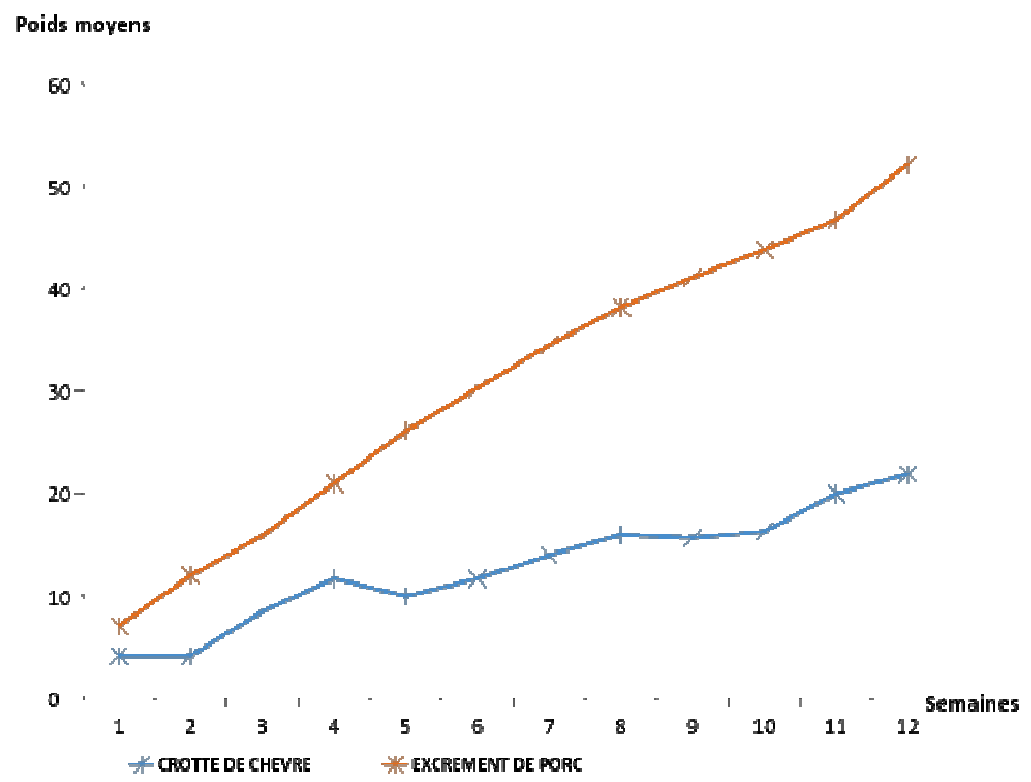

Figure 3 : Évolution pondérale du poids moyen des alevins selon le type de fertilisation au cours de 12 semaines

La croissance de deux courbes est différente du fait que la crotte des chèvres a un taux moins élevé en azote que les excréments des porcs, et plus l'étang est manipulé il y a toujours modification du rythme de croissance. Ce qui justifie que la courbe ne démontre pas une évolution linéaire croissante, au début elle est linéaire, puis croissante suivi d'une légère chute ainsi de suite. Par ailleurs, la courbe de croissance avec excréments de porcs, a une évolution croissante linéaire parce que les excréments de porcs sont riches en azote. Cette situation favorise l'augmentation de plancton qui sert à l'alimentation des alevins, ces derniers croissent plus vite (Figure 3). 


\section{INTERPRETATION DES RESULTATS}

Durant cette expérimentation l'accent a porté sur la croissance en poids de Oreochromis niloticus en fonction de la fertilisation des étangs. Les analyses physicochimiques des étangs expérimentaux ont donné une température moyenne de $24^{\circ} \mathrm{C}$, pendant toute la durée de l'expérience et les paramètres chimiques de l'eau ont donné :
(i) $\mathrm{Ca}: 23 \mathrm{mg} / \mathrm{l}$
(ii) $\quad \mathrm{Mg}: 12 \mathrm{mg} / \mathrm{l}$
(iii) $\quad \mathrm{NO}_{3}: 0,07 \mathrm{mg} / \mathrm{l}$
(iv) $\quad \mathrm{NO}_{2}: 3,53 \mathrm{mg} / \mathrm{l}$
(v) Carbonate : $302,2 \mathrm{mg} / \mathrm{l}$
(vi) Bicarbonate : $293 \mathrm{mg} / \mathrm{l}$
(vii) $\mathrm{pH}: 6,8$

Au regard de ces données, il s'est dégagé une conformité avec les normes recommandées par certains auteurs (Melard et Philippart, 2002 ; Melard, 1986 ; Kestemont et al., 2004) pour la qualité de l'eau de l'étang. Les facteurs génétiques permettent aussi une bonne croissance, le sexe, la souche, l'espèce et ce fait entraine une distribution asymétrique du poids (Wolh Farth et al, 1983). Cette distribution a été remarquée dans cette étude aussi, c'est pourquoi le poids moyen des individus est pris en compte lors des mensurations réalisées. En prélevant les résultats, les poids moyens suivants ont été obtenus pour les excréments des porcs : $21 \mathrm{~g}$ en février ; $38,16 \mathrm{~g}$ en mars et $52,1 \mathrm{~g}$ en avril, pour les crottes des chèvres ; $11,73 \mathrm{~g}$ en février, $15,96 \mathrm{~g}$ en mars, $31,9 \mathrm{~g}$ en

\section{CONCLUSION GENERALE ET RECOMMANDATIONS}

Durant cette expérimentation, l'accent a porté sur la croissance en poids de Oreochromis niloticus en fonction de la fertilisation des étangs à la ferme Naviundu à une période thermique bien déterminée allant du mois de Février au mois d'Avril 2011. L'étude a porté sur 90 alevins de Oreochromis niloticus dont le poids initial était de $2 \mathrm{~g}$ au début des expérimentations, qui ont été répartis dans 2 étangs fertilisés et un étang témoin. Au bout de 3 mois, les résultats réalisés ont donné le poids moyen de $52,1 \mathrm{~g}$ pour les alevins évoluant dans l'étang fertilisé au moyen des excréments des porcs, le poids moyen de $21,9 \mathrm{~g}$ pour les crottes des chèvres et $8,03 \mathrm{~g}$ pour l'étang

\section{REFERENCES}

Amakoe A, 2011. Formation en pisciculture : production d'alevins et gestion de ferme piscicole, Coordination Togolaise des Organisations Paysannes et de Producteurs Agricoles, Lomé avril et l'étang témoin nous a donné $3,2 \mathrm{~g}$ en février, $5,16 \mathrm{~g}$ en mars et $8,03 \mathrm{~g}$ en avril. Les résultats repris dans le tableau 5 montrent une différence des moyennes. En appliquant le test d'ANOVA, il ressort que les engrais ont un impact positif sur les poids des poissons et la différence entre les poids est très significative. Le test de TUKEY HSD montre que la différence entre les moyennes des différents engrais comparées deux à deux est significative. Le test de TAPPLY a fait ressortir une différence non significative des poids entre les semaines. En outre le test d'ANOVA démontre que l'influence de l'âge sur les poids est très significative. La croissance des poissons sur les quels s'est portée cette étude est dans les normes, cela a été observé dans ce sens qu'un: alevin au départ a présenté un poids moyen de $2 \mathrm{~g}$ et après 3 mois il a atteint un poids moyen de $52,1 \mathrm{~g}$ pour l'étang fertilisé au moyen des excréments des porcs; $21,9 \mathrm{~g}$ pour celui fertilisé au moyen des crottes des chèvres et $8,03 \mathrm{~g}$ pour l'étang témoin. Ces différences des moyennes observées concordent avec les résultats obtenus par F.A.O, (2003). Les déjections des porcs sont habituellement plus riches que celles des chèvres, du fait que les excréments des porcs contiennent un pourcentage élevé en azote qui constitue une source d'augmentation des planctons base de l'alimentation des poissons, pour leur bonne croissance (Depelchin et Depelchin, 1987 ; Hilbrands et Yzerman, 2004 ; Amakoe, 2011).

témoin. En faisant recours au test statistique et aux analyses de laboratoire, les résultats obtenus montrent une différence des moyennes des poids des alevins. II ressort que les engrais ont un impact positif sur les poids des poissons et la différence entre les poids est très significative en fonction des fertilisants utilisés. Ainsi, l'eau fertilisée tout comme le sol, voit sa production augmentée. Étant donné que les excréments de porc contiennent beaucoup d'azote, il serait recommandable aux pisciculteurs de recourir à ces excréments pour fertiliser leurs étangs en vue d'accroitre la production piscicole.

Anonyme 2007. Pisciculture, à la pêche aux revenus, SPORE: Le magazine du développement agricole et rural des pays ACP, No 132

Carballo E, Van eer A., Van schie T., Hilbrands A. 2008. La pisciculture à petite échelle en eau douce, 
Agrodok 15, 3e Edition Fondation Agromisa et CTA, Wageningen.

Chambres d'agriculture de lorraine 2005. Pisciculture d'étang, Référentiel Diversification

Depelchin J. et Depelchin A, 1987. Possibilités offertes par les élevages associés, Tropicultura, 5, 3, 113-117.

F.A.O, 2003 : Aquaculture production (1984-1986). F.A.O Fisheries circular, 815, FIDI, $106 \mathrm{p}$.

Feillet $P, 2014$ : les protéines de l'avenir, Futuribles, Vigie alimentation, Note d'analyse prospective, $n^{\circ} 4$

Hilbrands A et Yzerman C, 2004 La pisciculture à la ferme, Agrodok 21, 3e Edition, Fondation Agromisa, Wageningen.

Kestemont P., Micha J. C Et Falter V. 2004: les méthodes de production d'alevins de tilapias nilotica, Fundp, F.A.O, ROME.

Kestemont P, 2004: Alimentation et nutrition des poissons, DES-Aquaculture, CUD-Ulg-FUNDP, Namur.

Melard C, 1986. Les bases biologiques de l'élevage intensif du Tilapia du Nil. Cahiers d'Éthologie appliquée, Fasc 3, Vol 6, $224 p$, Liège.

Melard C. et Philippart J.C, 2002. Production des Tilapias en eau chaude industrielle en Belgique, Université de l'État à Liège, Liège.

Wolh farth W.G et HULATA G, 1983. Applied genetics of Tilapia, ICLAARM, Manilla, Philippine.

Wurtz-arlet J, 1980. La fertilisation des étangs, (In INRA: la Pisciculture en étang, éd. R. Billard, Paris). 